\title{
Electronic Band Structure and Photoemission States of $\mathrm{La}_{2 / 3} \mathrm{~Pb}_{1 / 3} \mathrm{MnO}_{3}$
}

\author{
W. TOKARZ ${ }^{a, *}$, M. KOWALIK $^{b, a}$, R. ZALECKI ${ }^{a}$ AND A. KOŁODZIEJCZYK ${ }^{a}$ \\ ${ }^{a}$ AGH University of Science and Technology, Department of Solid State Physics \\ al. A. Mickiewicza 30, 30-059 Cracow, Poland \\ ${ }^{b}$ Department of Physics, Rzeszów University of Technology, Powstańców Warszawy 6, 35-959 Rzeszów, Poland \\ We present the theoretical study of electronic and magnetic properties in a manganese perovskite \\ $\mathrm{La}_{2 / 3} \mathrm{~Pb}_{1 / 3} \mathrm{MnO}_{3}$. The calculations were carried out in frame of the first-principles density functional theory with \\ the general gradient approximation using the WIEN $2 \mathrm{~K}$ package. The $P 3 c 1$ crystal structure was taken from the \\ detailed X-ray diffraction data for the perovskite. The exact exchange energy was utilized for Mn $d$ electrons. \\ Density of states was determined by the modified tetrahedron method. As a result we get a valence band for \\ the spin up and down density of states with the gap for the latter of $1.85 \mathrm{eV}$. We noticed that conduction band \\ is mainly dominated by $d$ spin up manganese electrons and $\mathrm{Mn}\left(d_{x z}, d_{y z}\right)$ states have twice larger contribution \\ than $\left(d_{x^{2}-y^{2}}, d_{x y}\right)$. We attribute this to $\mathrm{Mn}-\mathrm{O}_{6}$ octahedral tilting. From the same reason $d_{3 z^{2}-r^{2}}$ state has no \\ contribution to the density of states at the Fermi energy $\left(E_{\mathrm{F}}\right)$. Comparison of total density of states with the \\ ultraviolet photoemission spectroscopy measurements shows similar features especially as far as the lead spectral \\ intensity from the $6 s$ electrons at about $-10 \mathrm{eV}$ is concerned. The calculated total magnetic moment per formula \\ unit is $3.66 \mu_{\mathrm{B}}$, the measured one $3.48 \mu_{\mathrm{B}} / \mathrm{f}$.u.
}

PACS: 71.20.-b, 71.55.Ak, 72.80.Ga, 75.47.Lx, 79.60.-i

\section{Introduction}

Manganites with general formula $\mathrm{ReAMnO}$, where Re are trivalent rare earth ions and $\mathrm{A}$ - divalent alkaline earth ions, exhibit a large variety of electric and magnetic properties due to complex interplay between charge and orbital degree of freedom of the mixed-valence Mn cations and the double-exchange (DE) interaction via oxygen modified by the Jahn-Teller distortion interaction and tilting of $\mathrm{Mn}-\mathrm{O}_{6}$ octahedrals [1]. Ferromagnetic (FM) ordering in $\mathrm{ReAMnO}_{3}$ has been attributed to the double-exchange interaction between the valence electron state of $\mathrm{Mn}^{3+}-\mathrm{O}^{2-}-\mathrm{Mn}^{4+}[2-5]$. The FM state with a large spin splitting of the conduction band into majority and minority sub-bands separated by the on-site Hund interaction is a consequence of DE and lattice distortion [6]. It was shown by [7] using the linear augmented plane wave method, that in $\mathrm{La}_{0.5} \mathrm{Ba}_{0.5} \mathrm{MnO}_{3}$ half metallic ground-state is theoretically predicted. Similar conclusion was made in the paper [8] for $\mathrm{La}_{1-x} \mathrm{Ca}_{x} \mathrm{MnO}_{3}$ system exploiting the local spin-density approximation with the general potential linearized augmented plane-wave [9]. Also in the paper [10] the theoretical calculations for the $\mathrm{La}_{0.75} \mathrm{~Pb}_{0.25} \mathrm{MnO}_{3}$ were presented. In our work we have focused our attention on theoretical electronic band structure for $\mathrm{La}_{2 / 3} \mathrm{~Pb}_{1 / 3} \mathrm{MnO}_{3}$ in $P 3 c 1$

\footnotetext{
* corresponding author; e-mail: tokarz@agh.edu.pl
}

crystal structure as a perspective application to the same compounds but substituted with $\mathrm{Fe}$ and their comparison with the electron photoemission spectrum and magnetic moments.

\section{Computational details}

The calculations were done in the WIEN 2K code [11] based on the density functional theory (DFT) $[12,13]$ and the generalized gradient approximation (GGA) [14]. The local exact exchange [15] in hybrid mode with [16] $\alpha=0.25$ fraction on $3 d$ Mn electrons was applied. Spinorbit interaction was not included. The optimum $k$-mesh size was examined by calculating total energy as a function of $k$ points number. $132 k$ points were chosen for volume, the lattice ratio $c / a$ and structure optimization and for final computation in reduced Brillouin zone. The self-consistent calculations were performed until a charge convergence was better than 0.0001e.

As a starting point the rhombohedral crystal structure $R-3 c$ was chosen, i.e. 167 according to International Tables for Crystallography (ITC) [17]. This structure was elongated twice in $z$ direction and one of the third $\mathrm{La}$ ion positions were substituted by $\mathrm{Pb}$. Next, a several structure optimizations were calculated. In the first the total energy minimum was achieved for $3 \%$ volume reduction. In the second step $c / a$ ratio was adjusted whilst keeping optimal volume constant. The final structure optimization was to move the atomic positions in 
order to minimize the calculated forces at the nuclei [18] for less than $5 \mathrm{mRy} / \mathrm{a} . u$. As a result $P 3 c 1$ structure (i.e. 158 in ITC) was received with the lattice constants: $a=5.3414 \AA$ and $c=13.254 \AA$ and atomic positions as follows: of $\mathrm{La}(2 / 3,1 / 3,0.8353),(1 / 3,2 / 3,0.1665)$, of $\mathrm{Pb}$ $(0,0,0.9997)$, of $\operatorname{Mn}(2 / 3,1 / 3,0.08405),(0,0,0.2502)$, $(1 / 3,2 / 3,0.9197)$ and of $\mathrm{O}(0.4676,0.4668,0.001285)$, $(0.7904,0.1309,0.67136),(0.1313,0.7915,0.3312)$. The results presented in the next paragraph was calculated based on these crystal settings.

\section{Results and their analysis}

Our calculations allow us to figure out the partial band structure separately for spin up and spin down. The presented density of states (DOS) results in Fig. 1 show some significant difference between two spin orientations just below the Fermi level $\left(E_{\mathrm{F}}\right)$. Whilst the spin up bands are continuous through $E_{\mathrm{F}}$ the spin down have a $1.85 \mathrm{eV}$ gap below the $E_{\mathrm{F}}$ in comparison to $0.67 \mathrm{eV}$ in the paper [10].Both band structures have a complex composition within the range from -8.2 to $-1.8 \mathrm{eV}$. This complexity is better visible in DOS plots presented in Fig. 2 where the partial contributions from $\mathrm{Mn}$ and $\mathrm{O}$ states are presented. The disparity from spin up and down states for $\mathrm{Mn}$ is noticeable. In this region parts from

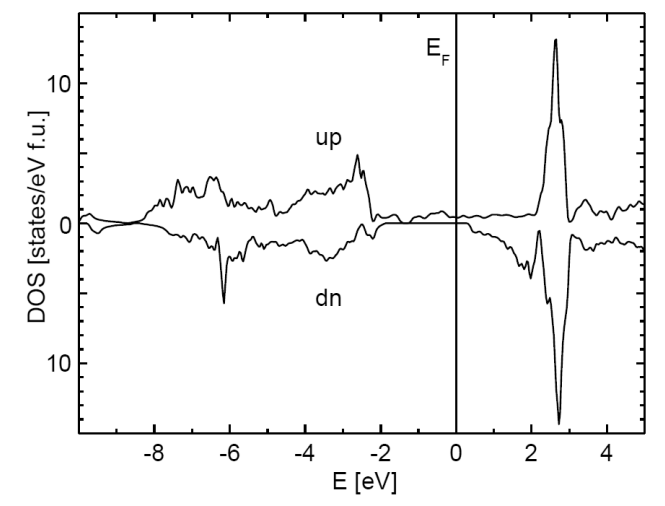

Fig. 1. Total DOS for spin up (up) and down (dn).

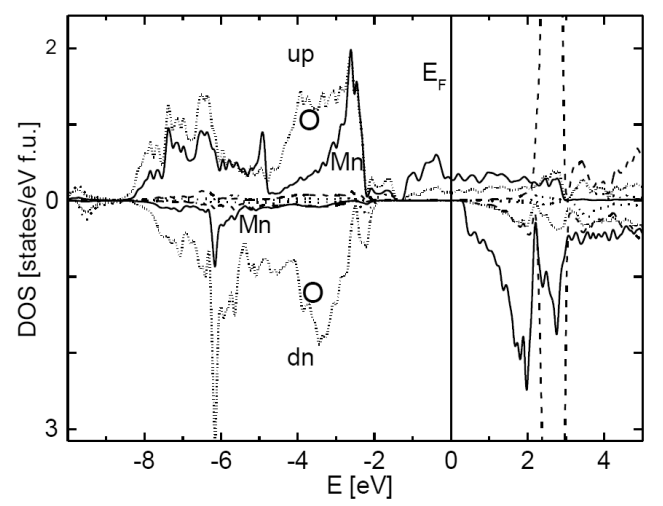

Fig. 2. Partial DOS of oxygen and manganese.

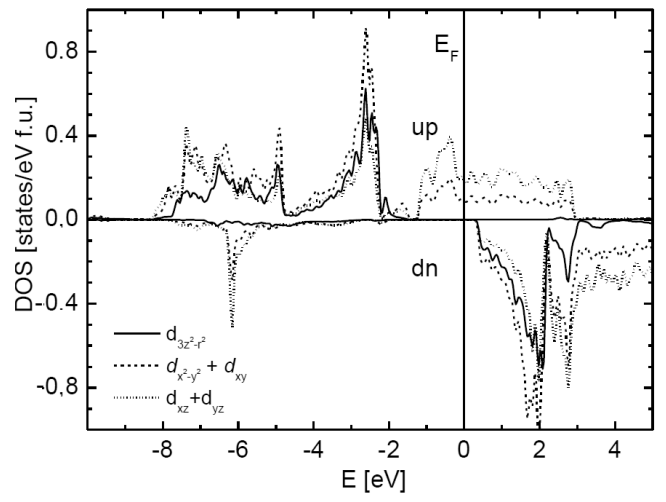

Fig. 3. DOS contributions from $d_{3 z^{2}-r^{2}},\left(d_{x y}, d_{x^{2}-y^{2}}\right)$ and $\left(d_{x z}, d_{y z}\right)$ manganese states.

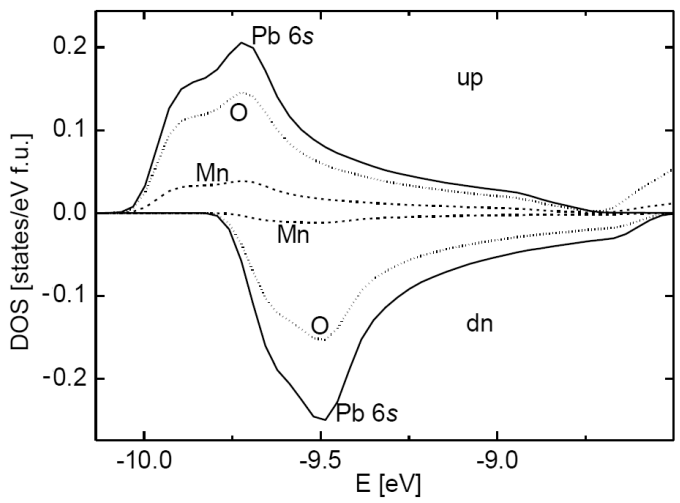

Fig. 4. Partial DOS at energy range from -10.2 to $\mathrm{La}{ }^{-7} \mathrm{eV}_{\mathrm{Pb}}$ are less than 0.12 states/eV f.u. In Fig. 3 it could be noticed that spin up density at $E_{\mathrm{F}}$ comes from the $\operatorname{Mn}\left(d_{x^{2}-y^{2}}, d_{x y}\right)$ and $\left(d_{x z}, d_{y z}\right)$ levels. In contrary, the $d_{3 z^{2}-r^{2}}$ contribution is about $1.3 \mathrm{eV}$ below $E_{\mathrm{F}}$. This density distribution is correlated with $\mathrm{Mn}-\mathrm{O}_{6}$ tilting and distortion. The $\mathrm{Mn}-\mathrm{O}_{6}$ octahedrals in the $P 3 c 1$ structure form the antiprisms with three oxygen in the bottom and top and $\mathrm{Mn}$ atom in the center. The symmetry of central $\mathrm{Mn}$ atom in octahedral position is $C_{3 v}$ [19]. In this symmetry the $d$ electrons energy levels are split into two levels: the first $e\left(d_{x z} d_{y z}\right)\left(d_{x y}, d_{x^{2}-y^{2}}\right)$ and the second $a\left(d_{3 z^{2}-r^{2}}\right)$. The magnetic field splits the $e$ states further into $\left(d_{x z}, d_{y z}\right)$ and $\left(d_{x y}, d_{x^{2}-y^{2}}\right)$. The last splitting is manifested at $E_{\mathrm{F}}$ by two times bigger densities from $\left(d_{x z} d_{y z}\right)$ than from $\left(d_{x y}, d_{x^{2}-y^{2}}\right)$. The $\mathrm{O}$ states also participate at $E_{\mathrm{F}}$. This outcome confirms the DE conductivity mechanism in this system between $\mathrm{Mn}-\mathrm{O}-\mathrm{M}$ via the oxygen $2 p$ states.

In our previous study [20] the half metallic gaps in this system were measured and estimated in room temperature to be from 1.7 to $2.0 \mathrm{eV}$ which is in very good agreement with these calculations. In the same work an open question was which states form the pronounced spectral density in the conduction band from $-12 \mathrm{eV}$ to $-9 \mathrm{eV}$. From our calculations we can conclude that this band is mainly composed of $\mathrm{Pb} 6 s$ states (cf. Fig. 4). The com- 


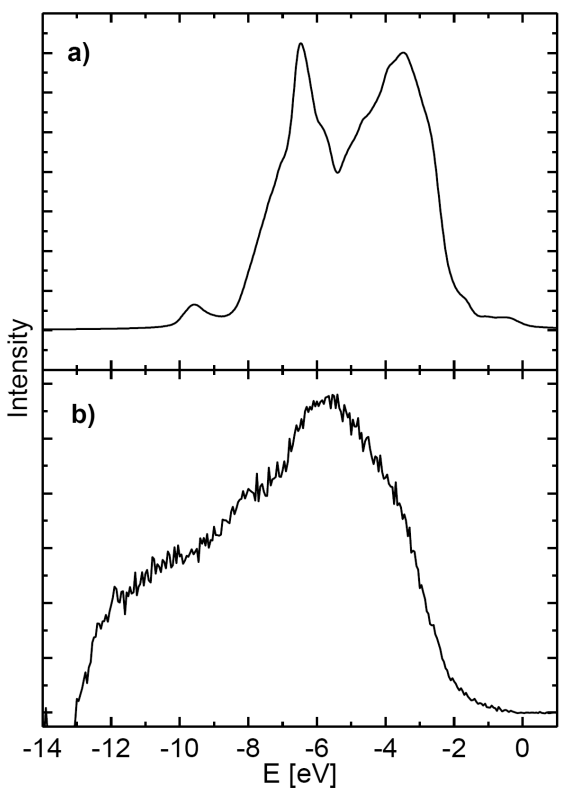

Fig. 5. Comparison of the calculated spectra (a) and the measured UPS at room temperature (b).

parison of calculated band electron photoemission spectrum and the ultraviolet electron photoemission (UPS) spectrum measured in our paper [20] is shown in Fig. 5. The theoretical spectrum was calculated taking into account the partial electronic densities of states weighted with atomic photoemission cross-section [21] for the photon energy $21.2 \mathrm{eV}$ ( $\mathrm{He}(\mathrm{I})$ source) and the experimental energy resolution was taken as the energy-dependent Lorentzian function with a half-width of about $0.3 \mathrm{eV}$. The calculated UPS spectrum is in qualitative agreement with the measured spectrum and proves the dominant role of Mn $3 d$ states at the Fermi energy. The calculated total magnetic moment per formula unit is $3.66 \mu_{\mathrm{B}} /$ f.u., while measured magnetic moment is $3.48 \mu_{\mathrm{B}} /$ f.u. [22].

\section{Conclusion}

We computed the electronic band structure of $\mathrm{La}_{2 / 3} \mathrm{~Pb}_{1 / 3} \mathrm{MnO}_{3}$ on energy optimizated $P 3 c 1$ crystal structure originated from $R-3 c 1$ structure [17]. The calculations show that the ground-state has a half-metallic character with $1.85 \mathrm{eV}$ gap for minority band. The valence band is mainly composed of manganese and oxygen states. At the $E_{\mathrm{F}}$ the manganese $d$ states, $\left(d_{x z} d_{y z}\right)$ and $\left(d_{x y}, d_{x^{2}-y^{2}}\right)$ and $\mathrm{O} 2 p$ have the strongest contribution. That confirms the DE conductivity mechanism in this system. The measured magnetic moment and the UPS spectrum are in qualitative agreement with the calculated ones.

\section{Acknowledgments}

This work was supported by the Polish Ministry of Science and Higher Education and its grants for Sci- entific Research. Authors thank to Dr. P. Stoch and Dr. J. Goraus for the important remarks about the WIEN $2 \mathrm{~K}$ calculations.

\section{References}

[1] E. Dagotto, Nanoscale Phase Separation and Colossal Magnetoresistance, Springer-Verlag, Berlin 2002.

[2] C. Zener, Phys. Rev. 82, 403 (1951).

[3] P.W. Anderson, H. Hasegawa, Phys. Rev. 100, 675 (1955).

[4] J. Goodenough, Phys. Rev. 100, 564 (1955).

[5] P.-G. de Gennes, Phys. Rev. 118, 141 (1960).

[6] P.G. de Gennes, J. Friedel, J. Phys. Chem. Solids 41, 71 (1958).

[7] N. Hamada, H. Sawada, K. Terakura, in: Spectroscopy of Mott Insulators and Correlated Metal, Eds. A. Fujimori, Y. Tokura, Springer-Verlag, Berlin 1995, p. 95.

[8] W.E. Pickett, D.J. Singh, Phys. Rev. B 53, 1146 (1996).

[9] D.J. Singh, Planewaves, Pseudopotentials, and the LAPW Method, Kluwer Academic, Boston 1994.

[10] A. Kowalczyk, A. Szajek, A. Slebarski, J. Baszynski, A. Winiarski, J. Magn. Magn. Mater. 217, 44 (2000).

[11] P. Blaha, K. Schwarz, G.K.H. Madsen, D. Kvasnicka, J. Luitz, WIEN2K: An Augmented Plane Wave and Local Orbitals Program for Calculating Crystal Properties, Vienna University of Technology, Vienna 2001.

[12] P. Hohenberg, W. Kohn, Phys. Rev. B 136, 864 (1964).

[13] W. Kohn, L.J. Sham, Phys. Rev. 140, 1133 (1965).

[14] J.P. Perdew, K. Burke, M. Ernzerhof, Phys. Rev. Lett. 77, 3865 (1996).

[15] J.C. Slater, Phys. Rev. 51, 195 (1937).

[16] F. Tran, P. Blaha, K. Schwarz, P. Novak, Phys. Rev. B 74, 155108 (2006).

[17] G. Gritzner, M. Koppe, K. Kellner, J. Przewoźnik, J. Chmist, A. Kołodziejczyk, K. Krop, Appl. Phys. A 81, 1491 (2005).

[18] R. Yu, D. Singh, H. Krakauer, Phys. Rev. B 43, 6411 (1991).

[19] P.W. Atkins, R. Friedman, Molecular Quantum Mechanics, 3rd ed., Oxford University Press, Oxford 1996.

[20] M. Kowalik, R. Zalecki, A. Kołodziejczyk, Acta Phys. Pol. A 117, 277 (2010).

[21] J.J. Yeh, I. Lindau, At. Data Nucl. Data Tables 32, 1 (1985).

[22] J. Przewoźnik, M. Kowalik, A. Kołodziejczyk, G. Gritzner, Cz. Kapusta, J. Alloys Comp. 497, 17 (2010). 